Volume 3

Issue 4 -- Cardiovascular Aging

Article 18

$11-11-2016$

\title{
An Interdisciplinary Process Change: Conversion of PICC Line Capping Solution From Heparin to Normal Saline
}

\author{
Angela Colella \\ Brandon Bodager \\ Frank Spexarth \\ Natalie McDonough \\ Deb Kastenholz \\ Paul Grebe
}

Follow this and additional works at: https://aah.org/jpcrr

Part of the Pharmacy Administration, Policy and Regulation Commons, and the Preventive Medicine Commons

\section{Recommended Citation}

Colella A, Bodager B, Spexarth F, McDonough N, Kastenholz D, Grebe P. An interdisciplinary process change: conversion of PICC line capping solution from heparin to normal saline. J Patient Cent Res Rev. 2016;3:239-40.

Published quarterly by Midwest-based health system Advocate Aurora Health and indexed in PubMed Central, the Journal of Patient-Centered Research and Reviews (JPCRR) is an open access, peer-reviewed medical journal focused on disseminating scholarly works devoted to improving patient-centered care practices, health outcomes, and the patient experience. 
2: Instructional procedures manual was created for 15 common procedures and provided to clinics. Phase 3: Postintervention survey was distributed after providers had access to procedures manual.

Results: Presurvey data revealed the majority of respondents felt neutral about their ability to perform procedures. However, residents felt more comfortable performing procedures than faculty. Postsurvey data revealed that a minority of respondents were aware of or used the procedures manual.

Conclusion: Our study demonstrated that a targeted intervention to improve the efficiency of procedure performance in the clinic setting was not well utilized. Therefore, a definitive conclusion about the ability of the intervention to improve the efficiency of procedure performance was not able to be made. Future areas of focus include better promotion of and ease of access to any selected intervention to improve efficiency of procedure performance.

\section{Prevalence of Prescription Opioid Abuse in Patients With Pain}

Samuel Godana, Ankoor Biswas, Nebiyu Biru, Abraham Getenet, Biana Leybishkis

\section{Department of Internal Medicine, Aurora Sinai Medical Center; Department of Internal Medicine, Aurora UW Medical Group}

Background: Studies showed that $20 \%$ to $30 \%$ of opioid analgesic drugs prescribed for chronic pain in the United States are misused, while the rate of opioid addiction is approximately $10 \%$. The study describes methods to identify high-risk behavior and forward recommendations to decrease opioid abuse.

Purpose: To assess prevalence and correlate multiple variables with opioid abuse and to forward appropriate recommendation to decrease the prevalence of opioid abuse in the primary care setting.

Methods: A prospective study was conducted at a primary health care clinic, Aurora Sinai Medical Center (Milwaukee, WI). Over 6 months, a total of 49 consecutive patients who take opioids were included in the study. Screener and Opioid Assessment for Patients with Pain-Revised (SOAPP-R), with a cutoff score of 18 (out of 24), is used to screen abuse/aberrant drug use. Additional variables were obtained by patient chart review. Data analysis was done using Minitab analysis software. Results: A total of 49 patients (23 males, 26 females) were included in the study. Mean age of patients was $45.7 \pm 7$ years; 22 (44.9\%) patients were on hydrocodone, $16(32.7 \%)$ on oxycodone, $7(14.3 \%)$ on tramadol, $3(6.1 \%)$ on fentanyl patch and $1(2 \%)$ on morphine and methadone. This means 33 $(66.3 \%)$ patients were on an opioid other than oxycodone. Of the 16 on oxycodone, 12 were male ( $52 \%$ of gender population) compared to 4 female ( $15.4 \%$ of gender population), $\mathrm{P}=0.013$. Five $(10.2 \%)$ patients had high SOAPP-R scores; 3 (13\%) of the males had a high SOAPP-R score compared to $2(7.7 \%)$ females. Regarding males on oxycodone, 3/12 (25\%) had high SOAPP-R scores; $1 / 4$ (25\%) females on oxycodone had a high SOAPP-R score. As age increased, the number of male patients using oxycodone trended upward. Older males on oxycodone had a higher SOAPP-R score compared to younger patients. No patient had prescriptions by more than one prescriber; 45 $(91.9 \%)$ patients had no pain contract. Urine drug screen was done in $11(22.5 \%)$ patients.

Conclusion: Prescription opioid drug abuse is not uncommon in primary care clinics. Use of the SOAPP-R, along with other appropriate patient evaluations, can help primary care providers identify high-risk behavior and decrease opioid abuse.

\section{An Interdisciplinary Process Change: Conversion of PICC Line Capping Solution From Heparin to Normal Saline}

Angela Colella, Brandon Bodager, Frank Spexarth, Natalie McDonough, Deb Kastenholz, Paul Grebe

Departments of Pharmacy, Radiology and Nursing, Aurora St. Luke's Medical Center, Aurora Health Care; AMG Imaging Services

Background: Heparin-induced thrombocytopenia (HIT) and HIT with thrombosis syndrome (HITTS) are serious conditions. Patients are at increased risk for developing HIT/HITTS with any exposure to heparin, even intravenous line flushes. Patients may be exposed to heparin multiple times each day when they have a peripherally inserted central catheter (PICC) that is flushed and capped with heparin. At Aurora Health Care, heparin is the standard capping solution for PICCs, but with a recent switch to positive pressurized caps, normal saline may be a capping option that reduces patient exposure to heparin.

Purpose: To reduce heparin exposure at a single hospital by replacing heparin with normal saline $(0.9 \%$ sodium chloride) as the standard PICC capping solution.

Methods: We implemented an interdisciplinary pilot process change and evaluated whether normal saline was noninferior to heparin for maintaining PICC patency. Primary outcome measurements of patency include alteplase use due to occlusion. Secondary objectives were any change in invasive line infection rates and cost comparisons. The baseline patency rate for the hospital was derived from data extracted through a retrospective chart review from October 2013 through October 2014.

Results: Our baseline patency rate was $65.42 \%(\mathrm{~N}=3,095)$; $33 \%$ of all PICC lines placed during the 12-month period were treated with alteplase. Patency rates during our pilot period were based on retrospective chart reviews of patients with PICC lines placed from February to May 2015. During this period, a total of 979 PICC lines were evaluated. Of these, $30.4 \%$ were treated with alteplase. This resulted in a pilot patency rate of $69.5 \%$, which is $4.1 \%$ greater than our baseline patency rate. Given the prespecified noninferiority margin of $5 \%$, our pilot demonstrates that normal saline is noninferior to heparin for maintaining PICC line patency when used with positive pressurized caps $(\alpha<0.05)$. Although number of infections increased $16 \%$, only $9 \%$ with line infections were part of our pilot. The estimated yearly cost of both capping solutions is equal, at approximately $\$ 3,960$ per our baseline 
usage data. By potentially avoiding a diagnosis of HIT/HITT in these patients, the hospital would realize an annualized savings of over $\$ 86,000$.

Conclusion: Normal saline has demonstrated historical noninferiority to heparin for maintaining PICC patency when used as the capping solution with positive pressurized caps. Similar results were found during our pilot.

\section{Cost-Effectiveness of Genomic-Based Warfarin Therapy}

John Weissert, Kourosh Ravvaz

\section{Aurora Research Institute}

Background: With over 40 years of demonstrated clinical efficacy, warfarin remains the world's most used pharmaceutical to prevent ischemic stroke in patients with atrial fibrillation (AF). However, warfarin has many challenges. Thus, despite known effectiveness, warfarin is a leading cause to druginduced morbidity and mortality. Over 50 different warfarin therapy protocols, including a number of pharmacogenomicbased (PG) protocols, with as many as 14 independent variables, have been developed to improve safety and efficacy, thereby reducing ischemic strokes and intracranial hemorrhages (ICH). Purpose: To conduct a preliminary cost-effectiveness study to determine the price point at which using warfarin PG dosing to prevent ischemic stroke and ICH would provide a neutral cost difference for the AF patient population at Aurora Health Care. Methods: Using a 15-year retrospective electronic medical record, we generated a large enough simulated AF population using Bayesian Network modeling to conduct a series of simulated warfarin therapies. We used five different PG and non-PG warfarin therapy protocols. The protocols with various levels of personalization used different PG and non-PG dosing algorithms for initial, adjustment and maintenance warfarin dosing. The simulation platform was able to predict daily international normalized ratio values and the rate of ischemic stroke and ICH in each simulated patient over 90 days for each of the five warfarin therapy protocols. Using nationwide estimates derived through literature review, we estimated the total cost of administering warfarin using the five different protocols and subsequent acute and 5-year care costs (adjusted according to annual Medical Care Component of the Consumer Price Index).

Results: In 2000-2015, a total of 48,006 patients, or $\sim 3,000$ patients annually, initiated warfarin therapy with a primary diagnosis of AF at Aurora. We found that Aurora's current best practice warfarin therapy protocol had the highest predicted costs for ICH, resulting in $\$ 163,462.55$ for acute care costs (averaged over 1,000 patients) and $\$ 171,279.60$ for 5-year ongoing care related to ICH. The current Aurora protocol also had the highest associated costs for ischemic stroke at $\$ 51,333.45$ for acute care and $\$ 58,507.21$ for 5-year ongoing care. In contrast, PG-protocol 3, which incorporated patient's genotype into the warfarin dosing protocol, had the lowest predicted acute care and ongoing costs associated with ICH at $\$ 119,823.78$ for acute care and $\$ 125,462.75$ for ongoing care.
For 1,000 AF patients initiating warfarin therapy in Aurora, switching to a PG protocol would save $\$ 55,299.94$ in acute care costs and $\$ 59,198.50$ in 5-year ongoing care.

Conclusion: At a cost of $\$ 59.20$ per patient, warfarin PG dosing is cost neutral. With substantial decreases in genotyping cost in recent years, it is likely this price is currently achievable. Therefore, although there is only incremental clinical benefit from warfarin PG dosing, it is likely cost-effective.

\section{Identifying Disparities in Colorectal Cancer Screening Rates in Milwaukee-Based Academic and Nonacademic Clinics}

Jasmine Wiley, Jonathan J. Blaza, Will Lehmann, Deborah Simpson, Jeffrey A. Stearns, Shelby L. Pischke, Tracy L. Greiten

Department of Family Medicine, Aurora Health Care; Department of Family Medicine, Aurora UW Medical Group; Academic Affairs, Aurora UW Medical Group; Business Intelligence, Aurora Health Care; Operational Informatics, Aurora Health Care

Background: The Institute for Healthcare Improvement's Triple Aim focuses on improving the patient's experience of care, improving population health and reducing the per capita cost of health care. Health care systems and providers continuously seek to improve quality of care through understanding what percentage of their patients are achieving quality-of-care standards for various indicators, including immunizations, tobacco cessation, asthma and cancer screening. As health care moves toward reimbursing for value-based care, deepening our understanding of patient population characteristics within each of these conditions is vital to continuous quality improvement. Purpose: To determine if there are race/ethnicity/age/preferred language (REAL) disparities in care to patients 50 years old or older who are eligible for colorectal cancer (CRC) screening in family medicine residency clinics.

Methods: A retrospective analysis of all patients eligible for CRC screening at two Milwaukee-based family medicine residency teaching clinics (referred to as FM1 and FM2) and nonacademic clinics in greater Milwaukee (NAC-MKE) during a 12-month period (December 2014 - November 2015) was undertaken in collaboration with health care system quality improvement specialists. Percentage of patients achieving CRC screening metric was reported by REAL and gender. As the ultimate goal was to identify subpopulations to target for improvement, categories with $\mathrm{N}<25$ were omitted and criterion for disparity within a category was defined as $>10 \%$.

Results: The largest CRC screening disparity was associated with age, with gaps ranging from $13 \%$ to $15 \%$ between populations $>65$ years old versus 50-54 years old: NAC-MKE (79\% vs 66\%), FM1 (81\% vs 68\%), and FM2 (80\% vs 65\%). CRC screening disparities varied by black/African-American race per location, 54\% at NAC-MKE and 67\% at FM2 (N $\leq 25$ at FM1). Other race, ethnicity and gender were $<10 \%$.

Conclusion: Per the Centers for Disease Control and Prevention, the African-American/black race has the highest 